\title{
Prevalence and determinants of persistent symptoms after treatment for Lyme borreliosis: study protocol for an observational, prospective cohort study (LymeProspect)
}

Hedwig D. Vrijmoeth ${ }^{1,3+}$, Jeanine Ursinus ${ }^{2,3^{*}+}$ (D), Margriet G. Harms ${ }^{3}$, Tizza P. Zomer ${ }^{5}$, Stefanie A. Gauw ${ }^{2}$, Anna D. Tulen ${ }^{3}$, Kristin Kremer ${ }^{3}$, Hein Sprong ${ }^{3}$, Hans Knoop ${ }^{4}$, Yolande M. Vermeeren ${ }^{5}$, Barend van Kooten ${ }^{5}$, Leo A. B. Joosten ${ }^{1+}$, Bart-Jan Kullberg ${ }^{1+}$, Joppe W. R. Hovius ${ }^{2+}$ and Cees C. van den Wijngaard ${ }^{3+}$

\begin{abstract}
Background: After antibiotic treatment of Lyme borreliosis, a subset of patients report persistent symptoms, also referred to as post-treatment Lyme disease syndrome. The reported prevalence of persistent symptoms varies considerably, and its pathophysiology is under debate. The LymeProspect study has been designed to investigate the prevalence, severity, and a wide range of hypotheses on the etiology of persistent symptoms among patients treated for Lyme borreliosis in the Netherlands.
\end{abstract}

Methods: LymeProspect is a prospective, observational cohort study among adults with proven or probable Lyme borreliosis, either erythema migrans or disseminated manifestations, included at the start of antibiotic treatment. During one year of follow-up, participants are subjected to questionnaires every three months and blood is collected repeatedly during the first three months. The primary outcome is the prevalence of persistent symptoms after treatment, assessed by questionnaires online focusing on fatigue (CIS, subscale fatigue severity), pain (SF-36, subscale pain) and neurocognitive dysfunction (CFQ). Potential microbiological, immunological, genetic, epidemiological and cognitive-behavioral determinants for persistent symptoms are secondary outcome measures. Control cohorts include patients with long-lasting symptoms and unconfirmed Lyme disease, population controls, and subjects having reported a tick bite not followed by Lyme borreliosis.

(Continued on next page)

\footnotetext{
* Correspondence: j.ursinus@amc.nl

${ }^{\dagger}$ Hedwig D. Vrijmoeth and Jeanine Ursinus are authors contributed equally to this work.

${ }^{\dagger}$ Leo A.B. Joosten, Bart-Jan Kullberg, Joppe W.R. Hovius and Cees C. van den

Wijngaard are shared senior authorship.

${ }^{2}$ Department of Internal Medicine, Division of Infectious Diseases \& Center

for Experimental and Molecular Medicine, Amsterdam UMC, University of Amsterdam, P.O. Box 22660, 1100, DD, Amsterdam, the Netherlands

${ }^{3}$ National Institute for Public Health and Environment (RIVM), Center for Infectious Disease Control, P.O. Box 1, 3720, BA, Bilthoven, the Netherlands Full list of author information is available at the end of the article
}

(c) The Author(s). 2019 Open Access This article is distributed under the terms of the Creative Commons Attribution 4.0 International License (http://creativecommons.org/licenses/by/4.0/), which permits unrestricted use, distribution, and reproduction in any medium, provided you give appropriate credit to the original author(s) and the source, provide a link to the Creative Commons license, and indicate if changes were made. The Creative Commons Public Domain Dedication waiver (http://creativecommons.org/publicdomain/zero/1.0/) applies to the data made available in this article, unless otherwise stated. 
(Continued from previous page)

Discussion: This article describes the background and design of the LymeProspect study protocol. This study is characterized by a prospective, explorative and multifaceted design. The results of this study will provide insights into the prevalence and determinants of persistent symptoms after treatment for Lyme borreliosis, and may provide a rationale for preventive and treatment recommendations.

Trial registration: NTR4998 (Netherlands Trial Register). Date of registration: 13 February 2015.

Keywords: Lyme disease, Borreliosis, Erythema Migrans, Borrelia, Persistent symptoms, Post-treatment Lyme disease syndrome, Study protocol

\section{Background}

Lyme borreliosis is caused by tick-borne Borrelia burgdorferi sensu lato genospecies. The incidence of tick bites and cases of Lyme borreliosis has increased substantially over the past decades. In the Netherlands, annually over one million people report a tick bite, and more than 25.000 cases of Lyme borreliosis are diagnosed [1-3]. The most common manifestation of Lyme borreliosis in Europe is erythema migrans, a red or bluish-red expanding skin lesion arising within days to weeks at the site of the tick bite. A minority of infected individuals develops disseminated Lyme borreliosis, of which Lyme neuroborreliosis, Lyme arthritis and acrodermatitis chronic atrophicans (ACA) are most common [4].

Although Lyme borreliosis generally responds well to antibiotic therapy, some patients report persistent symptoms after treatment. However, the prevalence of persistent symptoms in literature varies considerably, between 0 and 48\% [2, 5-17], and many of these studies lack proper controls. Case definition, Lyme borreliosis manifestation, follow-up, geographic location, and delay between onset of symptoms and treatment might explain the divergence in prevalence. Whereas the initial objective manifestation usually resolve, disabling symptoms such as fatigue, musculoskeletal pain and neurocognitive disturbances can last for months or even years, greatly affecting quality of life. When present for more than six months, post-treatment Lyme Disease Syndrome (PTLDS) is the term frequently used to describe these persistent symptoms $[9,18,19]$.

The prevalence as well as the underlying pathology of persistent symptoms after treatment for Lyme borreliosis are not clearly defined and under debate. Consequently, uniform guidelines on prevention and treatment of persistent symptoms are lacking. These uncertainties have led to great societal concern and dissatisfaction among patients. A broad range of hypotheses exists regarding the pathophysiology underlying persistent symptoms, ranging from microbiological, immunological, genetic, cognitive-behavioral, clinical, to epidemiological explanations. The LymeProspect study has been designed to test this broad range of hypotheses on persistent symptoms in a large cohort of patients with confirmed Lyme borreliosis. In this prospective, observational study, patients are followed from the start of antibiotic treatment during one year. This design allows us to assess the prevalence of persistent symptoms after treatment for Lyme borreliosis and to identify determinants of these symptoms. In this paper, we describe the study protocol in detail.

\section{Methods \\ Study design}

A multi-center prospective, observational cohort study with one year of follow-up is performed to determine the prevalence and severity of persistent symptoms after antimicrobial treatment for active Lyme borreliosis, and to assess determinants for development of these symptoms. Potential microbiological, immunological, genetic, clinical, cognitive-behavioral, and epidemiological determinants are assessed in patients with confirmed early or disseminated Lyme borreliosis. This study is a collaboration between the Dutch National Institute for Public Health and the Environment (RIVM, Bilthoven, the Netherlands) and the clinical expert centers for Lyme borreliosis at Amsterdam UMC (location AMC, University of Amsterdam, Amsterdam, the Netherlands), and Radboud university medical center (Radboudumc, Nijmegen, the Netherlands), in collaboration with the Lyme center Apeldoorn (Gelre Hospital, Apeldoorn, the Netherlands). The study has been approved by the medical ethics committee (METC) Noord-Holland (NL50227.094.14), and is conducted according to the principles of the Declaration of Helsinki.

\section{Study population}

This prospective study comprises adults with probable or proven Lyme borreliosis - either local infection or disseminated disease - in the Netherlands. Recruitment has started in April 2015. Recruitment of children has started in 2017 and results will be reported separately. Case definitions include clinical and laboratory criteria, and are largely based on the case definitions established by ESGBOR (Additional file 1: Table S1) [4]. Patients are included within 7 days after, but preferably before, initiation of antibiotic treatment. Patients with signs and 
symptoms attributed to a previous episode of Lyme borreliosis are excluded (Table 1). A separate cohort of patients with long-lasting symptoms attributed to Lyme borreliosis, without clinical and microbiological confirmation, is enrolled in the unconfirmed Lyme borreliosis control group. Inclusion criteria in this control group include symptoms (e.g. fatigue, cognitive complaints, myalgias and arthralgias) for more than 6 months with a severity above the Dutch norm score, as assessed by a combined questionnaire based on the CIS, SF-36 and CFQ as described below; negative serology (B. burgdorferi s.l. IgG ELISA or a combined IgM/IgG ELISA); and attribution of symptoms to Lyme borreliosis either because symptoms originated within one month after a documented tick bite, or because of a positive result of a non-recommended test (e.g., LTT or other commercially

Table 1 Inclusion and exclusion criteria

\begin{tabular}{|c|}
\hline Patients with confirmed Lyme borreliosis \\
\hline Inclusion criteria: \\
\hline $\begin{array}{l}\text { - Patients } \geq 18 \text { years with confirmed proven or probable early localized } \\
\text { or disseminated Lyme borreliosis manifestation as specified in } \\
\text { Additional file 1: Table S1; }\end{array}$ \\
\hline $\begin{array}{l}\text { - In case of an EM reported at www.tekenradar.nl, the EM has been } \\
\text { present }<3 \text { months and the clinical diagnosis has been confirmed } \\
\text { by the general practitioner (criteria for clinical diagnosis are } \\
\text { described in Additional file 1: Table S1); }\end{array}$ \\
\hline - Subjects live or stay on the mainland of the Netherlands. \\
\hline Exclusion criteria: \\
\hline $\begin{array}{l}\text { - Subjects unable to provide informed consent or not having a } \\
\text { sufficient command of the Dutch language; }\end{array}$ \\
\hline $\begin{array}{l}\text { - Subjects who started antibiotic treatment }>4 \text { days before inclusion } \\
\text { (for subjects included through the website www.tekenradar.nl) or }> \\
7 \text { days before inclusion (for subjects included through the clinical } \\
\text { expert centers for Lyme borreliosis); }\end{array}$ \\
\hline $\begin{array}{l}\text { - Subjects who have ongoing signs or symptoms attributed to a } \\
\text { previous episode of Lyme borreliosis. }\end{array}$ \\
\hline Patients with unconfirmed Lyme borreliosis \\
\hline Inclusion criteria: \\
\hline $\begin{array}{l}\text { - Myalgia, arthralgia, neuralgia, concentration disorders, cognitive } \\
\text { disturbances, with or without fatigue, present for } \geq 6 \text { months at } \\
\text { baseline }\end{array}$ \\
\hline $\begin{array}{l}\text { - Severity of symptoms assessed by the CIS, SF-36 and/or CFQ } \\
\text { questionnaire above the Dutch norm scores; }\end{array}$ \\
\hline $\begin{array}{l}\text { - Subjects have a negative serological test for B. burgdorferi s.l. (IgG } \\
\text { ELISA or C6 IgM/lgG ELISA), but have a history of an unconfirmed } \\
\text { suspicion of Lyme disease, based on }\end{array}$ \\
\hline $\begin{array}{l}\text { - a positive result for a non-recommended diagnostic test (e.g., } \\
\text { cellular tests, CD57 analysis, viable blood analysis, } \\
\text { bioresonance), or }\end{array}$ \\
\hline $\begin{array}{l}\text { - onset of disease symptoms that have started within one month } \\
\text { after a documented tick bite. }\end{array}$ \\
\hline Exclusion criteria: \\
\hline $\begin{array}{l}\text { - Subjects are unable to give informed consent or do not have } \\
\text { sufficient command of the Dutch language. }\end{array}$ \\
\hline
\end{tabular}

available cellular test, CD57 analysis) (Table 1). In addition, two other control cohorts are available for comparison. Firstly, a population control cohort consists of subjects randomly recruited nationwide, representing the general population. Data from patients and from these controls are matched by age, gender, geographical region, and month of enrollment. Secondly, a tick bite control cohort includes subjects who have reported a tick bite without having developed clinical evidence for a Borrelia infection at baseline or during follow-up. The same questionnaires are administered to individuals in all control cohorts and the prospective study patients during one year of follow-up.

\section{Recruitment, inclusion and follow-up of participants}

Recruitment, inclusion and follow-up of participants occur both online and at the clinical expert centers for Lyme borreliosis. The national website www.tekenradar. nl offers secure eligibility screening, inclusion and follow-up of participants with confirmed Lyme borreliosis after either self-registration or registration by a medical doctor (GP or specialist). After online enrollment, the participant's medical doctor is contacted to confirm the diagnosis. Written informed consent is obtained from eligible patients. After inclusion, blood collection tubes are delivered at the patient's home address by courier service, and blood is collected at a local blood draw service at baseline and after 6 weeks. Blood samples are sent overnight to the study laboratories and processed immediately upon arrival, to ensure the processing is completed within $24 \mathrm{~h}$ after blood collection.

In addition to online enrollment, study subjects as well as unconfirmed Lyme borreliosis controls are enrolled at the participating clinical expert centers. For these patients, blood samples are collected and processed immediately after inclusion. From a subset of patients with skin manifestations, skin biopsies are obtained after additional informed consent.

\section{Measurements}

For patients with confirmed Lyme borreliosis, standard demographical characteristics are reported at baseline, including gender, age, and highest educational level. Comorbidities are reported at baseline, and new medical diagnoses are evaluated during follow-up. Details on exposure to ticks, previous Lyme borreliosis episodes and the current Lyme borreliosis manifestation and treatment are collected. Patients with erythema migrans or other skin manifestations, who are included online are requested to upload a photograph of their skin lesion, for blinded evaluation by independent experts. Signs and symptoms are assessed at baseline and after 3, 6, 9 and 12 months, by online questionnaires on fatigue, pain and neurocognitive dysfunction (Table 2). Fatigue severity is 
Table 2 Data collection and measurements for all patients with confirmed Lyme borreliosis

\begin{tabular}{|c|c|c|c|c|c|c|c|c|}
\hline & Baseline & $\begin{array}{l}10 \\
\text { days }\end{array}$ & $\begin{array}{l}2 \\
\text { weeks }\end{array}$ & $\begin{array}{l}6 \\
\text { weeks }\end{array}$ & $\begin{array}{l}3 \\
\text { months }\end{array}$ & $\begin{array}{l}6 \\
\text { months }\end{array}$ & $\begin{array}{l}9 \\
\text { months }\end{array}$ & $\begin{array}{l}12 \\
\text { months }\end{array}$ \\
\hline Written information and informed consent & $x$ & & & & & & & \\
\hline Baseline characteristics & $x$ & & & & & & & \\
\hline Physical examination & $x^{a}$ & & & & $x^{a c}$ & $x^{\mathrm{ac}}$ & $x^{\mathrm{ac}}$ & $x^{a c}$ \\
\hline $\begin{array}{l}\text { Recording Lyme manifestation, treatment and concomitant } \\
\text { medication }\end{array}$ & $x$ & & $x^{b}$ & & $x$ & $x$ & $x$ & $x$ \\
\hline Recording adverse events & $x$ & & $x^{b}$ & & $x$ & $x$ & & $x^{a}$ \\
\hline \multicolumn{9}{|l|}{ Questionnaires } \\
\hline $\begin{array}{l}\text { Primary outcome: CIS (subscale fatigue), } \\
\text { SF-36 (subscale pain), CFQ }\end{array}$ & $x$ & & & & $x^{1}$ & $x$ & $x^{1}$ & $x$ \\
\hline $\begin{array}{l}\text { Clinical parameters: PHQ-15, SF-36 (subscale } \\
\text { physical functioning and subscale social } \\
\text { functioning), TiC-P (health-care use and } \\
\text { absenteeism of work) }\end{array}$ & $x$ & & & & $x$ & $x$ & $x$ & $x$ \\
\hline $\begin{array}{l}\text { Cognitive-behavioral parameters: brief IPQ, } \\
\text { CBRSQ, HADS, SES-F, PCS, IPAQ }\end{array}$ & $x$ & & & & $x$ & $x$ & & \\
\hline Comorbidities: TiC-P (co-morbidity list) & $x$ & & & & & & & $x$ \\
\hline Comorbidities: PREDIS & $x$ & & & & & & & \\
\hline \multicolumn{9}{|l|}{ Laboratory measurements } \\
\hline B. burgdorferi s.l. serology & $x$ & $x^{a c}$ & & $x$ & $x^{a c}$ & & & \\
\hline Serology other TBPs ${ }^{3}$ & $x^{2}$ & & & $x^{2}$ & & & & \\
\hline PCR B. burgdorferi s.l. and other TBPs ${ }^{3}$ (blood) & $x$ & & & $x$ & & & & \\
\hline Genome wide association studies & $x^{4}$ & & & & & & & \\
\hline Cytokine measurements in cell culture supernatants & X & & & X & & & & \\
\hline Gene expression micro-arrays on ex vivo stimulated PBMCs & $x^{5}$ & & & $x^{5}$ & & & & \\
\hline $\begin{array}{l}\text { Skin biopsies: culture, MLST, PCR B. burgdorferi s.l. and } \\
\text { other TBPs, gene-expression profiling }\end{array}$ & $x^{6}$ & & & & & & & \\
\hline
\end{tabular}

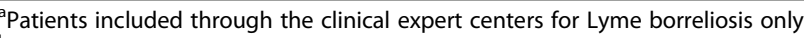

${ }^{b}$ Patients included through the website www.tekenradar.nl only

${ }^{c}$ These visits and laboratory measurements can be left out if patients are not able or not willing to. This is regarded as an allowed deviation from the protocol

${ }^{1} \mathrm{CIS}$ questionnaire only short version, to limit the burden for patients

${ }^{2}$ In cases (patients with persistent symptoms) and twice as much controls (patients without persistent symptoms), starting with the 6 weeks sample. If borderline or positive, the baseline sample will be tested as well

${ }^{3}$ Serology on Babesia spp., Rickettsia conorii, Anaplasma phagocytophilum (commercially available) and Borrelia miyamotoi (experimental). Quantitative PCR including Babesia spp., Rickettsia spp., Anaplasma phagocytophilum, Neoehrlichia mikurensis, as well as a pan relapsing fever Borrelia qPCR (if positive, a specific Borrelia miyamotoi qPCR will be performed)

${ }^{4}$ In the first consecutive included 600 patients with erythema migrans

${ }^{5}$ In a selection of patients, included through the clinical expert centers for Lyme borreliosis

${ }^{6}$ In patients with a skin manifestation included through the clinical expert centers for Lyme borreliosis (only after additional consent). Both the affected and contra-lateral side will be investigated

Abbreviations: (q)PCR (quantitative) polymerase chain reaction, TBPs tick-borne pathogens, PBMCs peripheral blood mononuclear cells, MLST Multilocus sequence typing. For the abbreviations of the various questionnaires, see the main text

assessed by the fatigue severity subscale of the Checklist Individual Strength (CIS) [20]. Scores range from 8 to 56 , and scores of 35 or higher reflect severe fatigue. The Medical Outcomes Survey Short Form-36 (SF-36) pain subscale is used to assess severity and impact of pain. Significant impairment due to pain is reflected by a score of 55 or lower, based on Dutch norm scores [21]. Neurocognitive functioning is assessed with the Dutch version of the Cognitive Failure Questionnaire (CFQ) [22]. Clinically significant complaints on neurocognitive functioning are reflected by a score of 44 or higher.
Clinical and cognitive-behavioral parameters are assessed by online questionnaires as well, including somatic symptoms (PHQ-15) [23], physical and social functioning (SF-36, subscales physical functioning and social functioning) [24], health care use, absenteeism from work, co-morbidity (adapted from TiC-P) [25], pre-existent symptoms (adapted from PREDIS) [26], illness perception (Brief IPQ) [27], cognitive and behavioral responses to symptoms (CBRSQ) [28], psychological distress (HADS) [29], self-efficacy with respect to pain and fatigue (SES, PCS) [30,31], and the 
level of physical activity (IPAQ) [32] (Table 2). Blood is collected at several time points for microbiological, immunological and genetic analyses (Table 2). For detection of B. burgdorferi s.l.-specific antibodies, a total Ig C6 ELISA (Immunetics, Boston, MA, USA) is performed on all samples, followed by IgM and IgG immunoblot analysis (Mikrogen GmbH, Neuried, Germany) for confirmation of C6 ELISA positive or borderline results. Furthermore, serology for other tick-borne pathogens is performed on at least all samples collected at six weeks. Immunofluorescence analysis (Focus Diagnostics, Cypress, CA, USA) is used for the detection of antibodies directed to Babesia spp., Rickettsia spp. and Anaplasma phagocytophilum. An IgM and IgG ELISA (TestLine Clinical Diagnostics, Brno, Czech Republic) for tick borne encephalitis (TBE) virus is conducted for patients with persistent symptoms and matched patients without persistent symptoms. Antibodies against B. miyamotoi are assessed by an experimental assay [33]. All blood samples are analyzed with (multiplex) real-time PCRs, based on various genes specific for B. burgdorferi s.l., $B$. miyamotoi, A. phagocytophilum, Candidatus Neoehrlichia mikurensis, spotted fever Rickettsia's, Bartonella spp., and a wide range of Babesia spp.. In patients with cutaneous Lyme borreliosis manifestations, skin biopsies are collected at baseline after additional consent, and assessed for B. burgdorferi s.l. culture and molecular detection of $B$. burgdorferi s.l. by PCR. Multilocus sequence typing (MLST) analysis is performed to type clinical B. burgdorferi s.l. isolates from skin biopsies.

Immunological assays include measurements of proand anti-inflammatory cytokines in cell culture supernatants of ex vivo stimulated whole blood and PBMCs, stimulated with RPMI (medium control), viable B. burgdorferi s.l. (mix of B. burgdorferi s.s., B. garinii and B. afzelii) at different multiplicities of infection (MOI), lipopolysaccharide (LPS), Pam3cys, and heat killed Candida albicans blastoconidia. Interleukin (IL)-1ß, IL-6, IL-1Ra and IL-10 produced after $24 \mathrm{~h}$ of exposure to these stimuli are measured with commercial ELISA kits. In a subset of patients, also data from pre-market and commercial cellular tests will be available. In addition, genome wide association studies (GWAS) are performed on DNA extracts from EDTA blood from the first consecutive 600 patients included with erythema migrans, to identify associations between single-nucleotide polymorphisms (SNPs) and the development of persistent symptoms, ex-vivo cytokine production profiles, or other determinants assessed in this study. Finally, in a selected subset of patients, gene expression profiling (microarray) is performed on skin biopsies from the lesion and contralateral healthy skin as well as on stimulated PBMCs.

From subjects in the unconfirmed Lyme borreliosis control group, blood is collected at baseline only. These controls are subjected to the same measurements as the confirmed patients, with the exception of genetic analysis and skin biopsies, whereas for the population control group and the tick bite control group only questionnaire data are obtained.

\section{Outcome measures and data analysis}

The primary outcome measure is the prevalence and severity of persistent symptoms in patients with confirmed Lyme borreliosis after treatment, assessed by standardized questionnaires on fatigue, pain and neurocognitive functioning. Persistent symptoms are defined as an impaired score for fatigue severity (CIS, subscale fatigue, score 35 or higher), pain (SF-36, subscale pain, score 55 or lower), or impaired neurocognitive functioning (CFQ, score 44 or higher), started within six months after treatment for confirmed Lyme borreliosis, and lasting for at least 6 months, as assessed by questionnaires administered at baseline, and 3, 6, 9 and 12 months after inclusion. Patients with scores exceeding the cutoff scores for fatigue, pain or neurocognitive functioning during at least six months are categorized as cases, i.e. Lyme borreliosis patients with persistent symptoms. To correct for baseline symptoms unrelated to Lyme borreliosis, the prevalence of symptoms in the population control cohort and tick bite control cohort are used. Variability and seasonality in scores on several questionnaires obtained during one year are compared between patients with confirmed Lyme borreliosis, the population control group and the tick bite control group. A separate analysis is performed on patients with persistent symptoms lasting less than 6 months.

Secondary outcome measures are potential microbiological, immunological, genetic, clinical, cognitive-behavioral, and epidemiological determinants of persistent symptoms. The association of these parameters with development of persistent symptoms is assessed by comparing results of confirmed Lyme borreliosis patients with persistent symptoms (cases) and without persistent symptoms (controls). Prediction rules for the risk and severity of persistent symptoms for individual patients will be developed. Determinants for persistent symptoms identified in patients with confirmed Lyme borreliosis are also assessed in the unconfirmed Lyme borreliosis control group in an exploratory manner.

\section{Sample size}

As mentioned above, the reported percentage of patients developing persistent symptoms after treatment for Lyme borreliosis varies widely from 0 to $48 \%$. For the present study, a prevalence of $5 \%$ is assumed, based on a retrospectively estimated annual incidence rate of persistent symptoms in patients with erythema migrans and disseminated Lyme borreliosis in the Netherlands [2]. At 
a $5 \%$ prevalence of persistent symptoms for a cohort of 2000 patients, a $25 \%$ lower proportion for a determinant can be detected in patients without symptoms, if present in at least $57 \%$ of patients with persistent symptoms (power $80 \%$, alpha 5\%). In a cohort of 1500 patients, a $25 \%$ lower proportion can be detected for a determinant that is present in at least $66 \%$ of cases with persistence of symptoms, and for a cohort of 1000 patients for one that is present in at least $77 \%$ of cases (power $80 \%$, alpha of $5 \%$ ). When the prevalence of persistent symptoms would be higher, the power to detect these differences increases.

\section{Discussion}

The LymeProspect study evaluates the prevalence and severity of persistent symptoms after treatment for Lyme borreliosis, and aims to identify determinants for these symptoms. This study is the first to apply a prospective design, investigating a broad range of hypotheses on the etiology of persistent symptoms in a large cohort of patients with confirmed Lyme borreliosis. Previous studies have described the prevalence of persistent symptoms attributed to Lyme borreliosis in the United States and in Europe. Several studies have reported associations between individual microbiological, immunological, genetic, clinical and cognitive-behavioral factors and persistent symptoms after treatment in specific groups of patients. Firstly, persistence of Borrelia infection after antibiotic treatment has been suggested as a cause of persistent symptoms after treatment [34], although this is difficult to detect with the current diagnostic tools, and randomized trials have not found beneficial effects of prolonged antibiotic treatment in patients with persistent symptoms [35-38]. Other microbiological hypotheses include culture positivity, infection by specific Borrelia burgdorferi s.l. genospecies or strains, and co-infection with other tick-borne pathogens [39-42], although there is no convincing evidence of long-term infection with other tick-borne pathogens in patients with persistent symptoms attributed to Lyme disease [43]. Secondly, an ongoing aberrant immune response and underlying genetic polymorphisms may play a role. This has been specifically well-studied in patients with antibiotic-refractory Lyme arthritis [44-46]. Furthermore, several cytokines or cytokine profiles have been related to persistence of symptoms after treatment for Lyme borreliosis [15, 47-50]. Thirdly, in a cognitive-behavioral model, the persistence of symptoms was related to beliefs and behavior of the patients in response to these symptoms. This model has been successfully applied to persistent symptoms in other conditions [51, 52], and previous studies have suggested that psychological factors could play a role in functional outcome in patients with Lyme borreliosis [10, 53]. Finally, clinical and epidemiological factors could be associated with persistence of symptoms, including age, clinical signs, co-morbidity, Lyme borreliosis manifestation, duration of symptoms before start of treatment, type of antibiotic treatment, and prior exposure to tick bites or Borrelia infection $[14,54]$.

However, conclusions of previous findings vary widely and are difficult to be generalized due to heterogeneity in case definitions, antibiotic treatment regimes, follow-up periods, control groups and primary outcomes. The strengths of the current study are its size, prospective approach, and explorative and multifaceted design, enabling a thorough analysis of the prevalence and determinants of persistent symptoms of Lyme borreliosis in the Netherlands. The use of validated questionnaires with norm scores and the availability of additional population and tick bite control groups will allow to accurately compare the prevalence and severity of symptoms in the patient cohort to the background prevalence. The control groups will also allow correction for development of non-specific symptoms over time and their seasonal changes.

The majority of participants are expected to be diagnosed with erythema migrans and to be enrolled online. To ascertain that subjects meet the inclusion criteria, medical information about the diagnosis is obtained from their physician in a standardized fashion, as well as a photograph of the skin lesions, which will be evaluated by independent experts. Serological evaluation at two time points could further contribute to the confirmation of active Lyme borreliosis. For patients who are enrolled through the clinical Lyme expertise centers and have consented to skin biopsy, B. burgdorferi s.l. culture and qPCR on skin biopsies for B. burgdorferi s.l. will enable further laboratory confirmation of the diagnosis.

Patients are encouraged to provide baseline blood samples before initiation of antibiotic therapy. To assess a potential effect of antibiotic treatment on microbiological and immunological endpoints, duration of prior treatment will be explored in sensitivity analyses. We have ruled out a significant effect of overnight shipping of blood samples on immunological outcomes in prior pilot studies (unpublished data).

The primary outcome measure has been defined as the presence of fatigue (CIS), pain (SF-36) and/or neurocognitive complaints (CFQ) presenting within six months after initial diagnosis and treatment, and lasting for at least six months. Fatigue, pain and neurocognitive complaints have been reported as major symptoms reported after Lyme borreliosis, and constitute the definition of PTLDS. Repeated assessment of symptoms every three months during one year of follow-up will enable to assess the time course of symptoms, and to detect disabling symptoms lasting shorter than six months as well. Furthermore, the impact of symptoms on general 
functioning, as measured by physical and social functioning, health-care use and absenteeism at work, is assessed. Subjects experiencing symptoms, or those preoccupied with Lyme borreliosis, could be more inclined to fill in questionnaires at baseline and to complete the follow-up, possibly leading to selection bias. This underscores the importance of the comparison of our primary outcomes in the patient cohort with the various control groups, since all three groups have the same scheme of follow-up and risk of bias.

In conclusion, the LymeProspect study is expected to provide additional insights into the prevalence and severity of persistent symptoms after antibiotic treatment for Lyme borreliosis. In addition, the study will identify determinants for persistent symptoms. These findings may lead to the development of prediction models for individual patients and may guide future research on preventive and therapeutic strategies of persistent symptoms after treatment for Lyme borreliosis.

\section{Additional file}

Additional file 1: Table S1. Clinical and laboratory criteria for inclusion of patients with confirmed Lyme borreliosis. Clinical and laboratory inclusion criteria for patients with confirmed Lyme borreliosis are described in detail. Criteria are largely based on case definitions published by Stanek et al. and alternative causes for symptoms should be excluded by the primary caregiver ${ }^{4}$. ${ }^{*}$ The CXCL-13 cut-off value is laboratory dependent. ${ }^{\#}$ As determined by neurological assessment or electromyogram. ^Preferably through synovial fluid puncture or synovium biopsy. ${ }^{\%}$ After a cardiologist has been consulted. (DOCX $17 \mathrm{~kb}$ )

\begin{abstract}
Abbreviations
(q)PCR: (quantitative) polymerase chain reaction; ACA: Acrodermatitis chronic atrophicans; CBRSQ: Cognitive Behavioural Responses to Symptoms Questionnaire; CFQ: Cognitive failure questionnaire; CIS: Checklist Individual Strength; CSF: Cerebrospinal fluid; DNA: Desoxyribo Nucleic Acid; EDTA: Ethylenediaminetetraacetic acid; ELISA: Enzyme-Linked Immuno Sorbent Assay; EM: Erythema migrans; GP: General practitioner; GWAS: Genome wide association studies; HADS: Hospital Anxiety and Depression Scale; IFA: Immunofluorescence assay; IL: Interleukine; IPAQ: International Physical Activity Questionnaire; IPQ: IIIness perception questionnaire; LPS: Lipopolysaccharide; LTT: Lymphocyte transformation test; MLST: Multilocus sequence typing; MOI: Multiplicity of infection; PBMC: Peripheral blood mononuclear cell; PBMCs: Peripheral blood mononuclear cells; PCS: Pain Catastrophizing Scale; PHQ: Patient Health Questionnaire; PTLDS: Post-treatment Lyme disease syndrome; s.l.: sensu lato; SES: Self-Efficacy Scale; SF-36: SF-36 Health Status Inventory; SNP: Single nucleotide polymorphism; spp.: species; TBE: Tick borne encephalitis; TBP: Tick-borne pathogens; Tic-P: Trimbos/iMTA questionnaire for Costs associated with Psychiatric IIIness
\end{abstract}

\section{Acknowledgements}

The authors acknowledge P. Assendelft, M.E. Baarsma, R. van Boxtel, J.I. Ersöz, E. Franz, I. Friesema, G.A. Oei, W. van Pelt, F.R. van de Schoor, A. van Vliet, H. van Weert, A. Wijmenga, and M. Zwerink, for their contribution to the design or implementation of the study.

\section{Funding}

This study is funded by the Netherlands Organisation for Health Research and Development (ZonMW) (project number 522001003) and by the Dutch Ministry of Health, Welfare and Sport (WWS). The study protocol has undergone peer-review by ZonMW. At the request of ZonMW, we made an adjustment of our study protocol by including a control group of patients with unconfirmed Lyme borreliosis. Both funders do not have a role in the collection, analysis and interpretation of data, as well as in writing the manuscript. The inclusion period for adults was extended to 01-10-2018, and the funding agency allowed continuation of the project until the end of 2019.

\section{Availability of data and materials}

Not applicable.

\section{Trial status}

Protocol version 5, 26-02-2018.

Date of first enrollment: 15-04-2015.

Recruitment completion date (adult participants): 01-10-2018.

Intended date of study completion (last adult participant, last time point): 30-09-2019.

\section{Authors' contributions}

HV and JU wrote the draft manuscript; HV, JU, MH, TZ, SG, AT, KK, HS, HK, YV, $\mathrm{BvK}, \mathrm{LJ}, \mathrm{BJK}, J \mathrm{H}$, and CvdW participated in the design of the study and the acquisition of data; all authors read and approved the final manuscript.

\section{Ethics approval and consent to participate}

This study has been approved by the medical ethics committee (METC) Noord-Holland (NL50227.094.14), and is conducted according to the principles of the Declaration of Helsinki. Written informed consent was obtained from all participants.

\section{Consent for publication}

Not applicable.

\section{Competing interests}

The authors declare that they have no competing interests.

\section{Publisher's Note}

Springer Nature remains neutral with regard to jurisdictional claims in published maps and institutional affiliations.

\section{Author details}

${ }^{1}$ Department of Internal Medicine and Radboud Center for Infectious Diseases, Radboud University Medical Center, P.O. Box 9101, 6500, HB, Nijmegen, the Netherlands. ${ }^{2}$ Department of Internal Medicine, Division of Infectious Diseases \& Center for Experimental and Molecular Medicine, Amsterdam UMC, University of Amsterdam, P.O. Box 22660, 1100, DD, Amsterdam, the Netherlands. ${ }^{3}$ National Institute for Public Health and Environment (RIVM), Center for Infectious Disease Control, P.O. Box 1, 3720, BA, Bilthoven, the Netherlands. ${ }^{4}$ Department of Medical Psychology, Amsterdam UMC, University of Amsterdam, P.O. Box 22660, 1100, DD, Amsterdam, the Netherlands. ${ }^{5}$ Lyme Center Apeldoorn, Gelre Hospital, P.O. Box 9014, 7300, DS, Apeldoorn, the Netherlands.

Received: 4 October 2018 Accepted: 3 April 2019

Published online: 15 April 2019

\section{References}

1. Hofhuis A, Harms M, van den Wijngaard C, Sprong H, van Pelt W. Continuing increase of tick bites and Lyme disease between 1994 and 2009 Ticks and tick-borne diseases. 2015;6(1):69-74.

2. Hofhuis A, Harms M, Bennema S, van den Wijngaard CC, van Pelt W. Physician reported incidence of early and late Lyme borreliosis. Parasit Vectors. 2015;8:161.

3. Hofhuis A, Bennema S, Harms M, van Vliet AJ, Takken W, van den Wijngaard CC, et al. Decrease in tick bite consultations and stabilization of early Lyme borreliosis in the Netherlands in 2014 after 15 years of continuous increase. BMC Public Health. 2016;16:425.

4. Stanek G, Fingerle V, Hunfeld KP, Jaulhac B, Kaiser R, Krause A, et al. Lyme borreliosis: clinical case definitions for diagnosis and management in Europe. Clinical microbiology and infection : the official publication of the European Society of Clinical Microbiology and Infectious Diseases. 2011; 17(1):69-79

5. Cerar D, Cerar T, Ruzic-Sabljic E, Wormser GP, Strle F. Subjective symptoms after treatment of early Lyme disease. Am J Med. 2010;123(1):79-86. 
6. Eikeland R, Mygland A, Herlofson K, Ljostad U. European neuroborreliosis: quality of life 30 months after treatment. Acta Neurol Scand. 2011;124(5): 349-54.

7. Nowakowski J, Nadelman RB, Sell R, McKenna D, Cavaliere LF, Holmgren D, et al. Long-term follow-up of patients with culture-confirmed Lyme disease. Am J Med. 2003;115(2):91-6.

8. Knudtzen FC, Andersen NS, Jensen TG, Skarphedinsson S. Characteristics and clinical outcome of Lyme Neuroborreliosis in a high endemic area, 1995-2014: a retrospective cohort study in Denmark. Clinical infectious diseases : an official publication of the Infectious Diseases Society of America. 2017;65(9):1489-95.

9. Aucott JN, Crowder LA, Kortte KB. Development of a foundation for a case definition of post-treatment Lyme disease syndrome. International journal of infectious diseases : IJID : official publication of the International Society for Infectious Diseases. 2013;17(6):e443-9.

10. Bechtold KT, Rebman AW, Crowder LA, Johnson-Greene D, Aucott JN. Standardized symptom measurement of individuals with early Lyme disease over time. Arch Clin Neuropsychol. 2017;32(2):129-41.

11. Eliassen $K E$, Hjetland $R$, Reiso $H$, Lindbaek $M$, Tschudi-Madsen $H$. Symptom load and general function among patients with erythema migrans: a prospective study with a 1-year follow-up after antibiotic treatment in Norwegian general practice. Scand J Prim Health Care. 2017;35(1):75-83.

12. Seltzer EG, Gerber MA, Cartter ML, Freudigman K, Shapiro ED. Long-term outcomes of persons with Lyme disease. JAMA. 2000;283(5):609-16.

13. Weitzner E, McKenna D, Nowakowski J, Scavarda C, Dornbush R, Bittker S, et al. Long-term assessment of post-treatment symptoms in patients with culture-confirmed early Lyme disease. Clinical infectious diseases : an official publication of the Infectious Diseases Society of America. 2015.

14. Ljostad U, Mygland A. Remaining complaints 1 year after treatment for acute Lyme neuroborreliosis; frequency, pattern and risk factors. Eur J Neurol. 2010;17(1):118-23.

15. Sjowall J, Fryland L, Nordberg M, Sjogren F, Garpmo U, Jansson C, et al. Decreased Th1-type inflammatory cytokine expression in the skin is associated with persisting symptoms after treatment of erythema migrans. PLoS One. 2011;6(3):e18220

16. Wormser GP, Weitzner E, McKenna D, Nadelman RB, Scavarda C Nowakowski J. Long-term assessment of fatigue in patients with cultureconfirmed Lyme disease. Am J Med. 2015;128(2):181-4.

17. Dersch R, Sommer H, Rauer S, Meerpohl JJ. Prevalence and spectrum of residual symptoms in Lyme neuroborreliosis after pharmacological treatment: a systematic review. J Neurol. 2015

18. van den Wijngaard CC, Hofhuis A, Harms MG, Haagsma JA, Wong A, de Wit $\mathrm{GA}$, et al. The burden of Lyme borreliosis expressed in disability-adjusted life years. Eur J Pub Health. 2015;25(6):1071-8.

19. Wormser G, Dattwyler R, Shapiro E, Halperin J, Steere A, Klempner M, et al. The clinical assessment, treatment, and prevention of Lyme disease, human granulocytic anaplasmosis, and babesiosis: clinical practice guidelines by the Infectious Diseases Society of America. Clin Infect Dis. 2006;43(9):1089-134.

20. Worm-Smeitink M, Gielissen M, Bloot L, van Laarhoven HWM, van Engelen BGM, van Riel P, et al. The assessment of fatigue: psychometric qualities and norms for the checklist individual strength. J Psychosom Res. 2017;98:40-6.

21. Aaronson N, Muller M, Cohen P, Essink-Bot M, Fekkes M, Sanderman R, et al. Translation, validation, and norming of the Dutch language version of the SF-36 health survey in community and chronic disease populations. J Clin Epidemiol. 1998;51(11):1055-68.

22. Ponds R, Van Boxtel M, Jolles J. De Cognitive Failure Questionnaire als maat voor subjectief cognitief functioneren. Tijdschrift voor Neuropsychologie. 2006;2:37-45.

23. Stegenga BT, Kamphuis MH, King M, Nazareth I, Geerlings MI. The natural course and outcome of major depressive disorder in primary care: the PREDICT-NL study. Soc Psychiatry Psychiatr Epidemiol. 2012;47(1):87-95.

24. Stewart JW, Quitkin FM, McGrath PJ, Rabkin JG, Markowitz JS, Tricamo E, et al. Social functioning in chronic depression: effect of 6 weeks of antidepressant treatment. Psychiatry Res. 1988;25(2):213-22.

25. Bouwmans C, De Jong K, Timman R, Zijlstra-Vlasveld M, Van der FeltzCornelis C, Tan Swan S, et al. Feasibility, reliability and validity of a questionnaire on healthcare consumption and productivity loss in patients with a psychiatric disorder (TiC-P). BMC Health Serv Res. 2013;13:217.

26. Heins MJ, Knoop H, Lobbestael J, Bleijenberg G. Childhood maltreatment and the response to cognitive behavior therapy for chronic fatigue syndrome. J Psychosom Res. 2011;71(6):404-10.
27. Broadbent E, Petrie KJ, Main J, Weinman J. The brief illness perception questionnaire. J Psychosom Res. 2006:60(6):631-7.

28. Skerrett TN, Moss-Morris R. Fatigue and social impairment in multiple sclerosis: the role of patients' cognitive and behavioral responses to their symptoms. J Psychosom Res. 2006;61(5):587-93.

29. Zigmond AS, Snaith RP. The hospital anxiety and depression scale. Acta Psychiatr Scand. 1983;67(6):361-70.

30. Sullivan MJL, Bishop SR, Pivik J. The pain catastrophizing scale: development and validation. Psychol Assess. 1995;7(4):524-32.

31. Nicholas MK. Self-efficacy and chronic pain. St Andrews: Annual Conference of the British Psychological Society; 1989.

32. Craig CL, Marshall AL, Sjostrom M, Bauman AE, Booth ML, Ainsworth BE, et al. International physical activity questionnaire: 12-country reliability and validity. Med Sci Sports Exerc. 2003;35(8):1381-95.

33. Koetsveld J, Kolyasnikova NM, Wagemakers A, Stukolova OA, Hoornstra D, Sarksyan DS, et al. Serodiagnosis of Borrelia miyamotoi disease by measuring antibodies against GlpQ and variable major proteins. Clinical microbiology and infection : the official publication of the European Society of Clinical Microbiology and Infectious Diseases. 2018.

34. Dattwyler R, Luft B, Kunkel M, Finkel M, Wormser G, Rush T, et al. Ceftriaxone compared with doxycycline for the treatment of acute disseminated Lyme disease. N Engl J Med. 1997;337(5):289-94.

35. Berende A, ter Hofstede HJ, Vos FJ, van Middendorp H, Vogelaar ML, Tromp $M$, et al. Randomized trial of longer-term therapy for symptoms attributed to Lyme disease. N Engl J Med. 2016;374(13):1209-20.

36. Cameron D. Severity of Lyme disease with persistent symptoms. Insights from a double-blind placebo-controlled clinical trial. Minerva Med. 2008; 99(5):489-96.

37. Klempner MS, Hu LT, Evans J, Schmid CH, Johnson GM, Trevino RP, et al. Two controlled trials of antibiotic treatment in patients with persistent symptoms and a history of Lyme disease. N Engl J Med. 2001;345(2):85-92.

38. Krupp LB, Hyman LG, Grimson R, Coyle PK, Melville P, Ahnn S, et al. Study and treatment of post Lyme disease (STOP-LD): a randomized double masked clinical trial. Neurology. 2003;60(12):1923-30.

39. Stupica D, Lusa L, Cerar T, Ruzic-Sabljic E, Strle F. Comparison of post-Lyme Borreliosis symptoms in erythema migrans patients with positive and negative Borrelia burgdorferi sensu lato skin culture. Vector borne and zoonotic diseases. 2011:11(7):883-9.

40. Strle F, Nadelman RB, Cimperman J, Nowakowski J, Picken RN, Schwartz I, et al. Comparison of culture-confirmed erythema migrans caused by Borrelia burgdorferi sensu stricto in New York state and by Borrelia afzelii in Slovenia. Ann Intern Med. 1999;130(1):32-6.

41. Jahfari S, Hofhuis A, Fonville M, van der Giessen J, van Pelt W, Sprong H. Molecular detection of tick-borne pathogens in humans with tick bites and erythema Migrans, in the Netherlands. PLoS Negl Trop Dis. 2016;10(10):e0005042.

42. Koetsveld J, Tijsse-Klasen E. Herremans T. Sprong H. Serological and molecular evidence for spotted fever group Rickettsia and Borrelia burgdorferi sensu lato co-infections in The Netherlands. Ticks and tick-borne diseases: Hovius JW: 2015.

43. Lantos PM, Wormser GP. Chronic coinfections in patients diagnosed with chronic Lyme disease: a systematic review. Am J Med. 2014;127(11):1105-10.

44. Londono D, Cadavid D, Drouin EE, Strle K, McHugh G, Aversa JM, et al. Antibodies to endothelial cell growth factor and obliterative microvascular lesions in the synovium of patients with antibiotic-refractory Lyme arthritis. Arthritis \& rheumatology. 2014;66(8):2124-33.

45. Steere AC, Falk B, Drouin EE, Baxter-Lowe LA, Hammer J, Nepom GT. Binding of outer surface protein a and human lymphocyte function-associated antigen 1 peptides to HLA-DR molecules associated with antibiotic treatment-resistant Lyme arthritis. Arthritis Rheum. 2003;48(2):534-40.

46. Shin JJ, Glickstein LJ, Steere AC. High levels of inflammatory chemokines and cytokines in joint fluid and synovial tissue throughout the course of antibiotic-refractory Lyme arthritis. Arthritis Rheum. 2007:56(4):1325-35.

47. Strle K, Sulka KB, Pianta A, Crowley JT, Arvikar SL, Anselmo A, et al. T-helper 17 cell cytokine responses in Lyme disease correlate with Borrelia burgdorferi antibodies during early infection and with autoantibodies late in the illness in patients with antibiotic-refractory Lyme arthritis. Clinical infectious diseases : an official publication of the Infectious Diseases Society of America. 2017:64(7):930-8.

48. Oosting M, ter Hofstede $H$, van de Veerdonk FL, Sturm P, Kullberg BJ, van der Meer JW, et al. Role of interleukin-23 (IL-23) receptor signaling for IL-17 responses in human Lyme disease. Infect Immun. 2011;79(11):4681-7. 
49. Aucott JN, Soloski MJ, Rebman AW, Crowder LA, Lahey LJ, Wagner CA, et al. CCL19 as a chemokine risk factor for posttreatment Lyme disease syndrome: a prospective clinical cohort study. Clinical and vaccine immunology : CVI. 2016:23(9):757-66.

50. Strle K, Stupica D, Drouin EE, Steere AC, Strle F. Elevated levels of IL-23 in a subset of patients with post-Lyme disease symptoms following erythema migrans. Clinical infectious diseases : an official publication of the Infectious Diseases Society of America. 2014;58(3):372-80.

51. Goedendorp M, Tack C, Steggink E, Bloot L, Bazelmans E, Knoop H. Chronic fatigue in type 1 diabetes: highly prevalent but not explained by hyperglycemia or glucose variability. Diabetes Care. 2014;37(1):73-80.

52. Knoop H, van Kessel K, Moss-Morris R. Which cognitions and behaviours mediate the positive effect of cognitive behavioural therapy on fatigue in patients with multiple sclerosis? Psychol Med. 2012;42(1):205-13.

53. Hassett AL, Radvanski DC, Buyske S, Savage SV, Gara M, Escobar Jl, et al. Role of psychiatric comorbidity in chronic Lyme disease. Arthritis Rheum. 2008; 59(12):1742-9.

54. Eikeland R, Mygland A, Herlofson K, Ljostad U. Risk factors for a nonfavorable outcome after treated European neuroborreliosis. Acta Neurol Scand. 2013;127(3):154-60.

Ready to submit your research? Choose BMC and benefit from:

- fast, convenient online submission

- thorough peer review by experienced researchers in your field

- rapid publication on acceptance

- support for research data, including large and complex data types

- gold Open Access which fosters wider collaboration and increased citations

- maximum visibility for your research: over $100 \mathrm{M}$ website views per year

At $\mathrm{BMC}$, research is always in progress.

Learn more biomedcentral.com/submissions 